\title{
Influence of Different Pigment Incorporation Methods on the Sorption and Solubility of Medical Silicones
}

\author{
Adhara Smith Nobrega ${ }^{1}$ Clóvis Lamartine de Moraes Melo Neto ${ }^{10}$ \\ André Pinheiro de Magalhães Bertoz ${ }^{1}$ André Luiz de Melo Moreno ${ }^{2}$ Marcelo Coelho Goiato ${ }^{1}$ \\ ${ }^{1}$ Department of Dental Materials and Prosthodontics, São Paulo State \\ University, School of Dentistry, Araçatuba, São Paulo, Brazil \\ 2 Department of Pediatric and Social Dentistry, São Paulo State \\ University, School of Dentistry, Araçatuba, São Paulo, Brazil

\begin{abstract}
Address for correspondence Marcelo Coelho Goiato, DDS, MSc, PhD, Department of Dental Materials and Prosthodontics, School of Dentistry, São Paulo State University, José Bonifácio Street, 1193 Araçatuba, São Paulo 16015-050, Brazil (e-mail: m.goiato@unesp.br).
\end{abstract}

Eur J Dent 2022;16:521-527.

\begin{abstract}
Keywords

- maxillofacial prosthesis

- solubility

- silicone elastomers

- A-2186

Objective The aim of this study is to verify the influence of three pigment incorporation methods (conventional, mechanical, and industrial) on the sorption and solubility of the MDX4-4210 and A-2186 silicones.

Materials and Methods The groups formed were based on the silicones used (A-2186 and MDX4-4210), intrinsic pigments (pink, bronze, and black), and pigment incorporation methods (conventional, mechanical, and industrial). The dimensions of all samples were 45 -mm diameter $(\varnothing) \times 1$-mm thickness. Readings were taken initially and after 1,008 hours of aging.

Statistical Analysis Three-way analysis of variance and the Tukey's test were performed $(\alpha=0.05)$.

Results For sorption and solubility, there was no difference between the incorporation methods for the A-2186 silicone, regardless of the pigment used $(p>0.05)$. For pink MDX4-4210, the industrial and mechanical methods showed higher values of sorption compared with the conventional method $(p<0.05)$. For bronze MDX4-4210, the industrial method showed a higher sorption value compared with the conventional and mechanical methods $(p<0.05)$. For black MDX4-4210, there was no difference between incorporation methods based on sorption ( $p>0.05)$. For pink MDX4-4210, the mechanical method showed a higher solubility value compared with the industrial and conventional methods $(p<0.05)$. For black MDX4-4210 and bronze MDX4-4210, there was no statistically significant difference between incorporation methods based on solubility $(p>0.05)$.

Conclusion Based on sorption and solubility, for the A-2186 silicone, the conventional, mechanical, and industrial methods of pigment incorporation were equivalent. For the MDX4-4210 silicone, its results of sorption and solubility were varied, and further studies are recommended.
\end{abstract}

published online

October 1, 2020
DOI https://doi.org/

$10.1055 / \mathrm{s}-0040-1716598$. ISSN 1305-7456. (c) 2020. The Author(s).

This is an open access article published by Thieme under the terms of the Creative Commons Attribution License, permitting unrestricted use, distribution, and reproduction so long as the original work is properly cited. (https://creativecommons.org/licenses/by/4.0/)

Thieme Medical and Scientific Publishers Pvt. Ltd., A-12, 2nd Floor, Sector 2, Noida-201301 UP, India 


\section{Introduction}

Facial defects can be the result of a congenital anomaly, surgical resection of a tumor, and trauma or a combination of these factors. ${ }^{1}$ Maxillofacial prostheses may be a treatment option for patients with orofacial defects. ${ }^{2}$ These prostheses have the function of protecting the tissues under it; in addition to returning facial aesthetics, high esteem, and quality of life for the patient. ${ }^{3}$ Silicone is a material widely used in the manufacture of maxillofacial prostheses. ${ }^{1-8}$

The degradation of the physical and mechanical properties of a silicone prosthesis is related to exposure to ultraviolet rays, air pollution, humidity, improper cleaning, and daily handling. ${ }^{1,3,4}$ The literature reports that the replacement of a silicone prosthesis occurs from 3 to 12 months after its manufacture due to its degradation. ${ }^{3,4,6}$ According to Mitra et al, one of the main reasons for replacing a maxillofacial prosthesis is due to the color change. ${ }^{6}$ In addition, Goiato et al reported that tear strength is one of the most important properties for the durability of a silicone prosthesis. ${ }^{5}$ Therefore, tearing of a silicone prosthesis can also be a reason for replacing this type of prosthesis. The MDX4-4210 and A-2186 silicones are examples of products widely used for the manufacture of maxillofacial prostheses. $3,4,6,7,9$

The water sorption and solubility tests evaluate the process of water gain and loss of soluble components to the environment. ${ }^{10}$ The sorption of a material represents the amount of water adsorbed on the surface and absorbed by the material body. ${ }^{10}$ Therefore, in the sorption process, both adsorption and absorption occur simultaneously. ${ }^{10}$ Solubility is represented by the solubilization of soluble compounds in a material. ${ }^{10}$ Therefore, any weight loss of a material is a measure of its solubility. ${ }^{10}$

A silicone prosthesis may be in frequent contact with saliva, sweat, and/or water (due to hygiene of the prosthesis or rain). ${ }^{2}$ Silicone is a material that can absorb water, saliva, and sweat ${ }^{2,11}$ and shows solubility. ${ }^{11}$ It is important to mention that these factors (absorption and solubility) can affect the physical, mechanical, and chemical properties of a polymer (e.g., silicone elastomer). ${ }^{2,11-16}$ In addition, Hulterström et al reported that if a prosthesis (e.g., silicone prosthesis) absorbs liquids to the point that it loses its original dimensions or shows solubility, the prosthesis may lose its functionality and appearance. ${ }^{11}$

The pigment incorporation method can influence the amount of bubbles that will be incorporated into a silicone. ${ }^{11}$ In the literature, three pigment incorporation methods can be observed, and these methods are classified as industrial, mechanical, and conventional (manual) ${ }^{4,5,8}$ In the literature, few studies have compared pigment incorporation methods; ${ }^{4,5,8}$ in addition, there are no studies comparing the influence of these methods on the sorption and solubility of medical silicones. Therefore, the objective of this study was to verify the influence of three pigment incorporation methods (industrial, mechanical, and conventional) on the sorption and solubility of the MDX4-4210 and A-2186 silicones. This study is the continuation of a previous study published in the European Journal of Dentistry. ${ }^{4}$

\section{Materials and Methods}

The A-2186 (Factor II, United States) and MDX4-4210 (Dow Corning Corporation Medical Products, United States) silicones were used in this study to manufacture the samples. - Fig. 1 shows the groups formed based on the silicones used (A-2186 and MDX4-4210), intrinsic pigments (black, bronze, and pink), and pigment incorporation methods (conventional, mechanical, and industrial). A total of 180 samples (90 samples for MDX4-4210 and 90 samples for A$2186)(n=10)$ with dimensions of $45-\mathrm{mm}$ diameter $(\varnothing) \times 1$ mm thickness ${ }^{7}$ were manufactured for the sorption and solubility tests (- Fig. 1). All samples were made by the same operator.

Bronze (Tan FI - 215, Factor II, United States), black (Black FI - 205, Factor II, United States) and medium pink (Orbital Colors, Brazil) pigments were used. The silicones and pigments were weighed on a digital analytical balance (Adventurer, Ohaus Corporation, United States). ${ }^{4,5}$ The bronze pigment corresponded to $0.2 \%$ of the weight of each silicone. ${ }^{4,5}$ The black pigment also corresponded to $0.2 \%$ of the weight of each silicone. ${ }^{4,5}$ For the pink pigment, the pigments that constituted it corresponded to $0.122 \%$ (yellow), $0.006 \%$ (black), $0.03 \%$ (red), and $0.6 \% \mathrm{TiO}_{2}$ (opacifier) of the weight of each silicone. ${ }^{4,5}$ With the exception of the opacifier which had a mineral origin, all other pigments used in this study had an organic origin. ${ }^{4,5}$

For the conventional method of incorporating the pigment to the silicone, the pigment was mixed with silicone manually., ${ }^{3,4}$ After the manipulation, the silicone was inserted into the matrix and the thickness was regularized with the aid of a metal spatula. ${ }^{3,4}$ The matrix was closed and submitted to $1,000 \mathrm{Kgf}$ for 10 minutes (Hydraulic pressMaxx, Essence Dental VH, Brazil). ${ }^{5}$ Samples remained confined within the matrix under controlled temperature with the surface exposed for 72 hours $\left(27 \pm 2^{\circ} \mathrm{C}\right)$ to complete polymerization of the material with release of the byproduct (formaldehyde).$^{3-5}$ After this period, the samples were carefully separated from the matrix. ${ }^{3}$

For the mechanical method of incorporating the intrinsic pigment to the silicone, the silicone was manually mixed to the pigment for 15 seconds, followed by a mechanical vacuum spatulation (15 psi) for 10 minutes at 425 RPM (Vacuum spatulator, Polidental, Brazil)., ${ }^{4,5}$ After this, the same steps of the conventional method were followed.

The industrial method of incorporating the pigment to the silicone was performed using a grinding machine (CHSG/3Roll Mill, Chemieland, China). ${ }^{4,5}$ The Deutsches Institut für Normung (DIN-53235) was used in this method. ${ }^{4,5}$ After this, the same steps of the conventional method were followed. ${ }^{4,5}$

The samples used in the sorption and solubility tests were submitted to a desiccation test according to the American Dental Association (ADA) - Specification ${ }^{12,17,18}$ before and after accelerated aging. For this procedure, the samples remained inside a dissector (Odontobrás, Brazil) containing silica gel and at a temperature of $37 \pm 2{ }^{\circ} \mathrm{C}$ for 23 hours. ${ }^{18}$ Subsequently, the samples were removed to a similar 

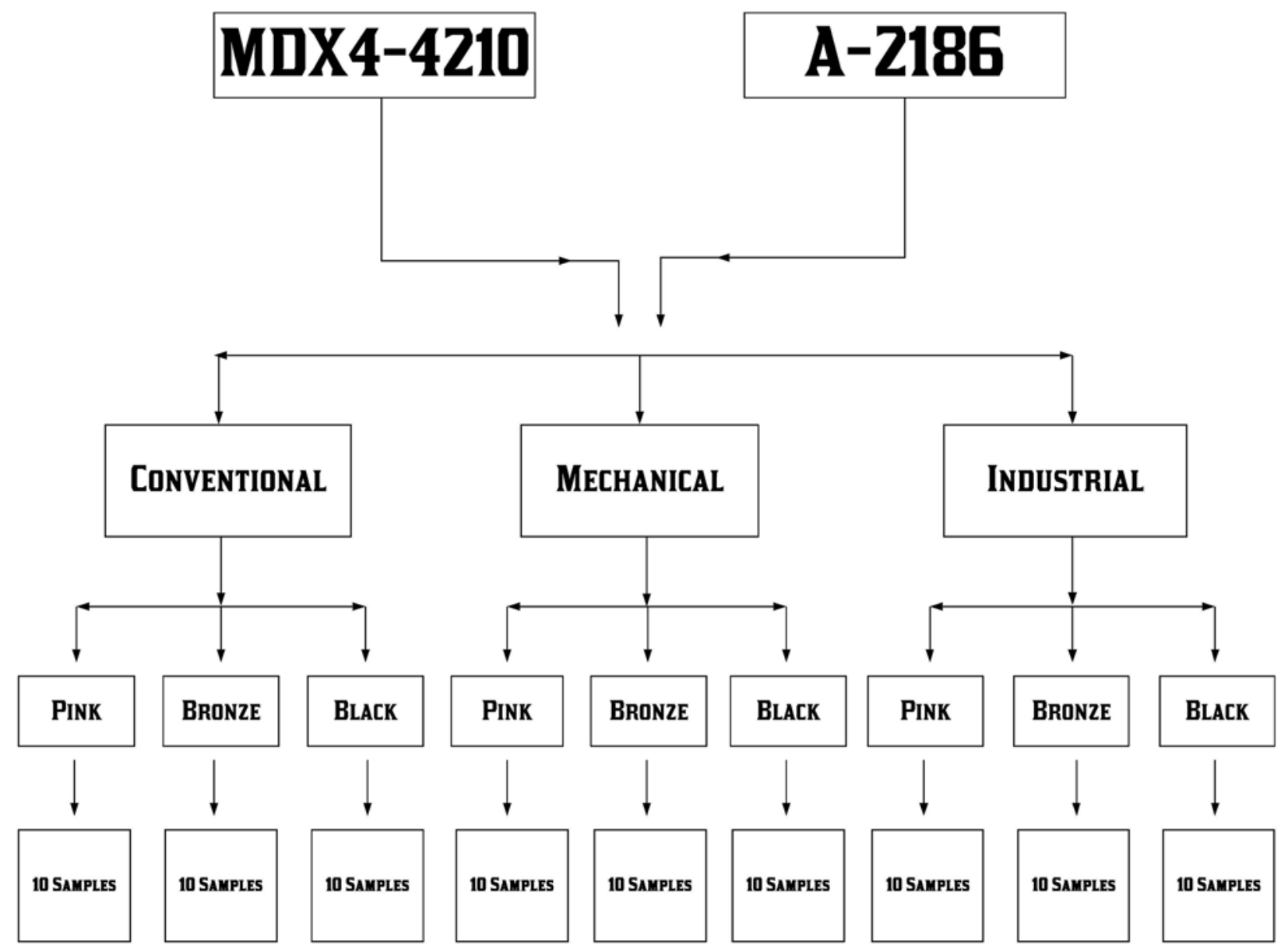

Fig. 1 Distribution of MDX4-4210 and A-2186 groups.

desiccator at room temperature $\left(23^{\circ} \mathrm{C}\right)$ for 1 hour and then weighed on a precision digital scale (BEL Equipamentos Analítico, Brazil). ${ }^{18}$ This cycle was repeated until the weight loss of each sample did not exceed more than $0.5 \mathrm{mg}{ }^{18}$ Therefore, the conditioned mass (W1) was reached when the difference between two successive readings did not exceed $0.5 \mathrm{mg}$. Posteriorly, the samples were submitted to the accelerated aging procedure, and they were weighed one more time (W2). Finally, the samples were submitted to a new dissection and final weighing (W3). The sorption and solubility were calculated according to the formulas: sorption $(\%)=(\mathrm{W} 2-\mathrm{W} 3) / \mathrm{W} 1 \times 100 ;$ solubility $\quad(\%)=(\mathrm{W} 1-$ W3 $) \times 100 .{ }^{17}$ The sorption and solubility tests were performed by the same operator.

The accelerated aging of the samples was performed in an aging chamber (Equilam, Brazil). This process was performed according to the American Society for Testing and Materials Designation G53-96. ${ }^{19}$ The lamps (UVB 313, 40 Watts, Equilam, Brazil) emitted ultraviolet B (UVB) light at a wavelength of $313 \mathrm{~nm} .^{5}$ Then, they were subjected to alternating periods of UVB light and distilled water condensation saturated with oxygen under conditions of heat and $100 \%$ humidity. ${ }^{3-5}$ Each aging cycle lasted 12 hours. ${ }^{3-5}$ In the first 8 hours, the temperature was maintained at $60 \pm 3^{\circ} \mathrm{C}$, and the
UVB light was imputed onto the samples. In the last 4 hours, the temperature was maintained at $45 \pm 3^{\circ} \mathrm{C}$ and a condensation period occurred without light. ${ }^{3-5}$ This test was performed for a total of 1,008 hours. $^{3-5}$

All data were analyzed using the Statistical Package for Social Sciences 20.0 (IBM Corp., United States). The ShapiroWilk statistical test was used to analyze the distribution of the numerical data. Three-way analysis of variance (ANOVA) and the Tukey's test were performed ( $\alpha=0.05)$.

\section{Results}

- Tables 1-4 show the Tukey's test for sorption and solubility.

- Table 1 shows the mean values (\%) and the standard deviation of sorption according to the incorporation method, pigment, and silicone brand. The pigment incorporation methods were compared based on the same pigment and silicone. For the A-2186 silicone, there was no statistically significant difference between the pigment incorporation methods based on sorption, regardless of the pigment used ( $p>0.05$; - Table 1). For the A-2186 silicone, based on sorption and the same pigment incorporation method, there was no statistically significant difference between the pigments used ( $p>0.05 ;-$ Table 1$)$. For the pink MDX4-4210 silicone, 
Table 1 Mean values (\%) and standard deviation of sorption of silicones according to the incorporation method, pigment, and silicone brand

\begin{tabular}{|l|l|l|l|l|}
\hline \multirow{2}{*}{ Silicone } & Pigment & \multicolumn{4}{l|}{ Incorporation method } \\
\cline { 2 - 5 } & & Conventional & Mechanical & Industrial \\
\hline \multirow{3}{*}{ A-2186 } & Bronze & $0.07(0.04)^{\mathrm{Aa}}$ & $0.04(0.02)^{\mathrm{Aa}}$ & $0.05(0.02)^{\mathrm{Aa}}$ \\
\cline { 2 - 5 } & Black & $0.06(0.04)^{\mathrm{Aa}}$ & $0.06(0.02)^{\mathrm{Aa}}$ & $0.04(0.01)^{\mathrm{Aa}}$ \\
\cline { 2 - 5 } & Pink & $0.05(0.01)^{\mathrm{Aa}}$ & $0.05(0.03)^{\mathrm{Aa}}$ & $0.07(0.03)^{\mathrm{Aa}}$ \\
\hline \multirow{3}{*}{ MDX4-4210 } & Bronze & $0.06(0.03)^{\mathrm{Aa}}$ & $0.08(0.03)^{\mathrm{Aa}}$ & $0.12(0.05)^{\mathrm{Ab}}$ \\
\cline { 2 - 5 } & Black & $0.07(0.02)^{\mathrm{Aa}}$ & $0.06(0.02)^{\mathrm{Aa}}$ & $0.04(0.03)^{\mathrm{Ba}}$ \\
\cline { 2 - 5 } & Pink & $0.05(0.04)^{\mathrm{Aa}}$ & $0.12(0.11)^{\mathrm{Bb}}$ & $0.12(0.07)^{\mathrm{Ab}}$ \\
\hline
\end{tabular}

Note: Tukey's test with a level of significance of $5 \%$. Different uppercase letters in the column denote a statistically significant difference $(p<0.05)$. Different lowercase letters in the row denote a statistically significant difference $(p<0.05)$.

the industrial and mechanical methods showed higher values of sorption compared with the conventional method $(p<0.05$; - Table 1). For bronze MDX4-4210, the industrial method showed a higher sorption value compared with the conventional and mechanical methods ( $p<0.05$; - Table 1). For black MDX4-4210 silicone, there was no difference between incorporation methods based on sorption ( $p>0.05$; - Table 1). For the MDX4-4210 silicone, based on sorption and the same pigment incorporation method: the pink MDX4-4210 manufactured by the mechanical method showed a higher sorption value than the bronze MDX4-4210 and black MDX4-4210 manufactured by the same method $(p<0.05)$; the black MDX4-4210 manufactured by the industrial method showed a lower sorption value than the bronze MDX4-4210, and pink MDX4-4210 manufactured by the same method ( $p<0.05 ;-$ Table 1$)$.

- Table 2 shows the mean values (\%) and the standard deviation of sorption according to the silicone and pigment incorporation method, and regardless of the pigment. For the A-2186 silicone, there was no difference between the incorporation methods based on sorption $(p>0.05)$ ( - Table 2 ). For the MDX4-4210 silicone, the sorption values were higher in the mechanical and industrial methods when compared with the conventional method ( $p<0.05$; - Table 2 ). The mechanical and industrial methods of the MDX4-4210 silicone showed higher sorption values than the mechanical and industrial methods of the A-2186 silicone $(\boldsymbol{p}<0.05$; - Table 2$)$.

- Table 3 shows the mean values (\%) and the standard deviation of solubility according to the incorporation method, pigment, and silicone brand. The pigment incorporation methods were compared based on the same pigment and silicone (-Table 3). For the A-2186 silicone, there was no difference between the pigment incorporation methods based on solubility, regardless of the pigment used ( $p>0.05$; - Table 3 ). For the A-2186 silicone, based on solubility and the same pigment incorporation method, there was no statistically significant difference between the pigments used ( $p>0.05$; - Table 3 ). For the pink MDX4-4210 silicone, the mechanical method showed a higher solubility value compared with the industrial and conventional methods $(p<0.05$; - Table 3 ). For black MDX4-4210 and bronze MDX4-4210, there was no statistically significant difference between incorporation methods based on
Table 2 Mean values (\%) and standard deviation of sorption of the different silicones according to the incorporation method, regardless of the pigment color

\begin{tabular}{|l|l|l|}
\hline \multirow{2}{*}{ Incorporation method } & \multicolumn{2}{|l|}{ Silicone } \\
\cline { 2 - 3 } & A-2186 & MDX4-4210 \\
\hline Conventional & $0.06(0.03)^{\mathrm{Aa}}$ & $0.06(0.03)^{\mathrm{Aa}}$ \\
\hline Mechanical & $0.05(0.03)^{\mathrm{Aa}}$ & $0.09(0.07)^{\mathrm{Bb}}$ \\
\hline Industrial & $0.05(0.02)^{\mathrm{Aa}}$ & $0.09(0.06)^{\mathrm{Bb}}$ \\
\hline
\end{tabular}

Note: Tukey's test with a level of significance of $5 \%$. Different uppercase letters in the column denote a statistically significant difference $(p<0.05)$. Different lowercase letters in the row denote a statistically significant difference $(p<0.05)$.

solubility ( $p>0.05$; - Table 3 ). For the MDX4-4210 silicone, based on solubility and the same pigment incorporation method: the bronze MDX4-4210 manufactured by the conventional method showed a higher solubility value than the black MDX44210 and pink MDX4-4210 manufactured by the same method $(p<0.05)$; the pink MDX4-4210 manufactured by the mechanical method showed a higher solubility value than the black MDX4-4210 manufactured by the same method ( - Table 3 ).

- Table 4 shows the mean values (\%) and the standard deviation of solubility according to the silicone and pigment incorporation method, and regardless of the pigment. For the A-2186 silicone, there was no difference between the incorporation methods based on solubility ( $p>0.05$; - Table 4$)$. For the MDX4-4210 silicone, the sorption value was higher in the mechanical method compared with the industrial method $(p<0.05 ;-$ Table 4$)$. The industrial method of the A-2186 silicone showed a higher solubility value than the industrial method of the MDX4-4210 ( $p<0.05$; - Table 4).

\section{Discussion}

When a polymer is exposed to a humid environment, two processes occur simultaneously: plasticizers and other soluble components are leached out of the polymer (solubility), and water is absorbed by the polymer. ${ }^{12,13}$ The amount of water absorbed by a polymer depends a lot on its chemical structure. ${ }^{14}$ The water absorption is mainly caused by the 
Table 3 Mean values (\%) and standard deviation of solubility of silicones according to the incorporation method, pigment, and silicone brand

\begin{tabular}{|l|l|l|l|l|}
\hline \multirow{2}{*}{ Silicone } & Pigment & \multicolumn{4}{l|}{ Incorporation method } \\
\cline { 2 - 5 } & & Conventional & Mechanical & Industrial \\
\hline \multirow{3}{*}{ A-2186 } & Bronze & $1.60(0.87)^{\mathrm{Aa}}$ & $1.52(0.33)^{\mathrm{Aa}}$ & $2.23(0.85)^{\mathrm{Aa}}$ \\
\cline { 2 - 5 } & Black & $1.35(0.57)^{\mathrm{Aa}}$ & $1.75(1.26)^{\mathrm{Aa}}$ & $2.76(4.01)^{\mathrm{Aa}}$ \\
\cline { 2 - 5 } & Pink & $1.85(1.00)^{\mathrm{Aa}}$ & $1.31(0.66)^{\mathrm{Aa}}$ & $1.78(0.66)^{\mathrm{Aa}}$ \\
\hline \multirow{3}{*}{ MDX4-4210 } & Bronze & $1.87(0.59)^{\mathrm{Aa}}$ & $0.31(0.21)^{\mathrm{Aa}}$ & $0.74(0.41)^{\mathrm{Aa}}$ \\
\cline { 2 - 5 } & Black & $0.11(0.22)^{\mathrm{Ba}}$ & $2.49(5.28)^{\mathrm{Bb}}$ & $0.60(0.21)^{\mathrm{Aa}}$ \\
\cline { 2 - 5 } & Pink & $0.35(0.16)^{\mathrm{Ba}}$ & $0.75)^{\mathrm{Aa}}$ & 0.53 \\
\end{tabular}

Note: Tukey's test with a level of significance of $5 \%$. Different uppercase letters in the column denote a statistically significant difference ( $p<0.05$ ). Different lowercase letters in the row denote a statistically significant difference $(p<0.05)$.

Table 4 Mean values (\%) and standard deviation of solubility of the different silicones according to the incorporation method, regardless of the pigment color

\begin{tabular}{|l|l|l|}
\hline \multirow{2}{*}{ Incorporation method } & \multicolumn{2}{|l|}{ Silicone } \\
\cline { 2 - 3 } & $\mathrm{A}-2186$ & MDX4-4210 \\
\hline Conventional & $1.60(0.83)^{\mathrm{Aa}}$ & $0.78(0.87)^{\mathrm{ABa}}$ \\
\hline Mechanical & $1.53(0.84)^{\mathrm{Aa}}$ & $1.49(3.10)^{\mathrm{Aa}}$ \\
\hline Industrial & $2.26(2.35)^{\mathrm{Aa}}$ & $0.62(0.50)^{\mathrm{Bb}}$ \\
\hline
\end{tabular}

Note: Tukey's test with a level of significance of $5 \%$. Different uppercase letters in the column denote a statistically significant difference $(p<0.05)$. Different lowercase letters in the row denote a statistically significant difference $(p<0.05)$.

polar properties of the polymer molecules. ${ }^{15}$ According to Arima et al and Garcia-Fierro and Aleman, because water interacts with the polymer chains, it can produce some of the following effects in this order: (1) reorientation and chain displacement, that is, reversible loosening or effective plasticization of the structure; (2) solvation or reversible rupture of weak interchain bonds; and (3) irreversible disruption of the polymer matrix (microvoids). ${ }^{16,20}$ Therefore, based on these situations, water absorption and solubility can alter the physical (e.g., color), mechanical (e.g., tear strength and hardness), and chemical properties of a polymer; ${ }^{2,11-16}$ in addition to causing dimensional changes in this type of material. ${ }^{12,16}$ Thus, sorption and solubility can affect the durability of a silicone prosthesis.

According to Goiato et al 2019, the period of 1,008 hours of accelerated aging represents 1 year of constant use of a silicone prosthesis by a patient. ${ }^{5}$ In the accelerated aging process, UVB rays are emitted in the silicone samples. Most polymers contain aromatic rings and $C=C$ bonds in their structures. ${ }^{4}$ Aromatic rings and $\mathrm{C}=\mathrm{C}$ bonds can absorb UVB rays during accelerated aging. ${ }^{4}$ According to Nobrega et al and Nobrega et al, when a polymer molecule absorbs UVB rays, this energy promotes instability in the molecular structure. $^{3,4}$ The excess energy can be transmitted by excitation from one molecule to another, allowing the first molecule to regain its stability. ${ }^{3,4}$ In this way, affected groups can return to their original state by releasing energy in the form of longer wavelengths such as visible light or heat., ${ }^{3,4}$
However, when this excess energy is released, a photochemical degradation occurs, contributing to molecule deterioration. $^{3,4}$ Tetteh et al can also explain a situation of the degradation of a silicone. ${ }^{21}$ Tetteh et al reported that the weathering can induce changes in physical, mechanical, and chemical characteristics of a polymer (e.g., silicone elastomer). ${ }^{21}$ The degradation of a polymer due to weathering is a result of a photo-oxidative attack (a combined action of oxygen and sunlight) on the chemical structure of this material. ${ }^{21}$ The photo-oxidative degradation causes an initial formation of free radicals, reaction of free radicals with oxygen, production of polymer oxy- and peroxy- radicals, and secondary polymer radicals, resulting in chain scission. ${ }^{21}$ In addition, a reaction of different free radicals with each other can result in crosslinking. ${ }^{21}$ It is also important to mention that a crosslinking can occur due to the formation of bonds between existing monomers or bonds between chains.21 Based on these situations, it is possible that the degradation of a silicone due to aging can change the sorption and solubility rates of this type of material. Therefore, accelerated aging is justified to simulate the clinical degradation of a silicone.

In this study, for the A-2186 silicone, when the incorporation methods were compared, there was no statistically significant difference based on sorption or solubility, regardless of the pigment used (- Tables 1-3). The MDX4-4210 silicone did not show the same result pattern when compared with the A-2186 silicone (-Tables 1-3). This difference in the pattern of results between the two silicones may have occurred due to a possible higher filler loading and molecular weight of the dimethylsiloxane polymer from the A-2186 silicone when compared with the MDX4-4210 silicone. ${ }^{5,22}$ In addition, a difference between these silicones based on the polar properties of their molecules may also have influenced their results. ${ }^{5}$

In most situations, MDX4-4210 silicone showed greater sorption than the A-2186 silicone $(p<0.05$; - Table 2$)$. On the other hand, A-2186 silicone showed a trend towards greater solubility when compared with the MDX4-4210 silicone (-Table 4). Perhaps as mentioned earlier, the possible different characteristics between these two silicones may have caused these situations. 
Based on the results of sorption and solubility of the MDX4-4210 silicone (-Tables 1-4), the mechanical and industrial methods did not always show the best results (lower sorption and solubility). In this study, there were situations in which the conventional method was superior (lower sorption and solubility) when compared with the mechanical and industrial methods. It was expected that the mechanical and industrial methods would always show the best results or at least equivalent results when compared with the conventional method (due to a probable situation of less of bubbles in the material, that is, less incorporation of "humidity reservoirs"). Based on these situations, it is possible to assume that, in addition to the intrinsic characteristics of the MDX4-4210 silicone that may have influenced its sorption and solubility results, the results of this study also suggest that an intrinsic pigmentation can influence the MDX4-4210 silicone, causing unexpected (as mentioned earlier) and varied sorption and solubility results (absence of a clear pattern between the results of the MDX4-4210 groups) (-Tables 1-3). This can be suggested, for example, by the fact that in the sorption evaluation, black MDX4-4210 did not show a difference between the incorporation methods ( $p>0.05)$; however, this situation was different for pink MDX4-4210 and bronze MDX4-4210, because there was a significant difference between incorporation methods in some cases $(p<0.05 ;-$ Table 1$)$. In the solubility assessment, only pink MDX4-4210 showed a significant difference between incorporation methods $(p<0.05)$; thus, this corroborates the fact that an intrinsic pigmentation can generate an influence on the MDX4-4210 silicone $^{9}$ (-Table 3). In addition, for the MDX4-4210 silicone, there were statistically significant differences between pigmentations in several cases (in each method evaluated individually), based on the values of sorption and solubility; thus, this also corroborates the fact that the MDX4-4210 silicone can be influenced by an intrinsic pigmentation ${ }^{9}$ ( - Tables 1-3). Therefore, presumably, an intrinsic pigmentation and the intrinsic characteristics of the MDX4-4210 silicone may have a greater influence on the sorption and solubility results of this silicone than a pigment incorporation method. It is worth mentioning that the sorption and solubility results of the A-2186 silicone were not affected by pigmentations or pigment incorporation methods. (-Tables 1-4).

Nobrega et al evaluated color and dimensional stability using the pigment incorporation methods used in this study (conventional, mechanical and industrial), for the manufacture of the MDX4-4210 and A-2186 silicones. Although all results were clinically acceptable for dimensional and color stability after aging regardless of the incorporation method used, the mechanical and industrial methods showed the best statistical results in general (lower color and dimensional change than the conventional method). ${ }^{4}$ In addition, Goiato et al showed an equivalence between the industrial and mechanical methods based on tear strength. ${ }^{5}$ Despite these situations, the industrial method can have a higher cost and require more time for the manufacture of a prosthesis when compared with the mechanical method. ${ }^{4,5}$ Another important aspect is that according to Hatamleh and Watts, mixing the base and catalyst of a silicone under vacuum (mechanical method) can reduce the amount of bubbles incorporated in this material compared with the conventional method (manual). ${ }^{8}$ It is important to mention that air bubbles inside a silicone prosthesis can affect its elasticity, elongation, tear resistance, and aesthetics. ${ }^{8}$ In this study for sorption and solubility, the three methods were equivalent based on A-2186 silicone in all cases. For the MDX4-4210 silicone, the results were often the opposite of what would be expected. It is worth mentioning that the results of previous studies ${ }^{4,5,8}$ regarding pigment incorporation methods cannot be disregarded in this study. Therefore, the mechanical method would be the best option for the manufacture of silicone prostheses.

A major limitation of this study is that in the literature there are no clinical acceptability criteria (ADA or International Organization for Standardization) for sorption or solubility of silicones used in maxillofacial prostheses. Therefore, the interpretation of the results of this study was more difficult, because it was not possible to know which percentage change for sorption or solubility after the aging of a silicone prosthesis, would be clinically acceptable. Another limitation is that few studies have compared pigment incorporation methods.

\section{Conclusion}

Based on sorption and solubility for the A-2186 silicone, the conventional, mechanical, and industrial methods of pigment incorporation were equivalent. For the MDX4-4210 silicone, its results of sorption and solubility were varied, and further studies are recommended.

Conflict of Interest

None declared.

\section{References}

1 Cevik P, Yildirim-Bicer AZ. Effect of different types of disinfection solution and aging on the hardness and colour stability of maxillofacial silicone elastomers. Int J Artif Organs 2017. Doi: 10.5301/ijao.5000659

2 Rai SY, Guttal SS. Effect of intrinsic pigmentation on the tear strength and water sorption of two commercially available silicone elastomers. J Indian Prosthodont Soc 2013;13(01):30-35

3 Nobrega AS, Andreotti AM, Moreno A, Sinhoreti MA, Dos Santos DM, Goiato MC. Influence of adding nanoparticles on the hardness, tear strength, and permanent deformation of facial silicone subjected to accelerated aging. J Prosthet Dent 2016;116(04): 623-629.e1

4 Nobrega AS, Malavazi EM, Melo Neto CLM, et al. Influence of different pigment incorporation methods on color, dimensional stability, and detail reproduction of silicones. Eur J Dent 2019;13 (03):399-404

5 Goiato MC, Nobrega AS, Freitas da Silva EV, et al. Tear strength analysis of MDX4-4210 and A-2186 silicones with different intrinsic pigments incorporated by mechanical and industrial methods. Int J Dent 2019;2019:2573095

6 Mitra A, Choudhary S, Garg H, H. G J. Maxillofacial prosthetic materials- an inclination towards silicones. J Clin Diagn Res 2014; 8(12):ZE08-ZE13 
7 Lai JH, Wang LL, Ko CC, DeLong RL, Hodges JS. New organosilicon maxillofacial prosthetic materials. Dent Mater 2002;18(03):281-286

8 Hatamleh MM, Watts DC. Porosity and color of maxillofacial silicone elastomer. J Prosthodont 2011;20(01):60-66

9 Malavazi Marrega E, dos Santos DM, de Moraes Melo Neto CL, de Caxias FP, da Silva EVF, Bannwart LC, et al. Influence of Different Pigmentations and Accelerated Aging on the Hardness and Tear Strength of the A-2186 and MDX4-4210 Silicones. Int J Dent 2020; 2020:8492091

10 Saini R, Kotian R, Madhyastha P, Srikant N. Comparative study of sorption and solubility of heat-cure and self-cure acrylic resins in different solutions. Indian J Dent Res 2016;27(03):288-294

11 Hulterström AK, Berglund A, Ruyter IE. Wettability, water sorption and water solubility of seven silicone elastomers used for maxillofacial prostheses. J Mater Sci Mater Med 2008;19(01):225-231

12 Braden M, Wright PS. Water absorption and water solubility of soft lining materials for acrylic dentures. J Dent Res 1983;62(06): 764-768

13 Garg A, Shenoy KK. A comparative evaluation of effect on water sorption and solubility of a temporary soft denture liner material when stored either in distilled water, 5.25\% sodium hypochlorite or artificial saliva: an in vitro study. J Indian Prosthodont Soc 2016;16(01):53-62

14 Scherillo G, Petretta M, Galizia M, La Manna P, Musto P, Mensitieri $G$. Thermodynamics of water sorption in high performance glassy thermoplastic polymers. Front Chem 2014;2:25
15 Polat TN, Karacaer O, Tezvergil A, Lassila LV, Vallittu PK. Water sorption, solubility and dimensional changes of denture base polymers reinforced with short glass fibers. J Biomater Appl 2003;17(04):321-335

16 Arima T, Murata H, Hamada T. The effects of cross-linking agents on the water sorption and solubility characteristics of denture base resin. J Oral Rehabil 1996;23(07):476-480

17 Goiato MC, Silva EVFD, Medeiros RA, et al. Effect of nonthermal plasma on the properties of a resinous liner submitted to aging. J Prosthet Dent 2018;119(03):397-403

18 Council on Dental Materials and Devices Revised American Dental Association specification no. 12 for denture base polymers. J Am Dent Assoc 1975;90(02):451-458

19 ASTM G53-96, Practice for Operating Light- and Water-Exposure Apparatus (Fluorescent UV-Condensation Type) for Exposure of Nonmetallic Materials (Withdrawn 2000), ASTM International, West Conshohocken, PA, 1996. Accessed August 5, 2020 at: https://www.astm.org/DATABASE.CART/WITHDRAWN/G53.htm

20 Garcia-Fierro JL, Aleman JV. Sorption of water by epoxide prepolymers. Macromolecules 1982;15:1145-1149

21 Tetteh S, Bibb RJ, Martin SJ. Mechanical and morphological effect of plant based antimicrobial solutions on maxillofacial silicone elastomer. Materials (Basel) 2018;11(06):925

22 Lai JH, Hodges JS. Effects of processing parameters on physical properties of the silicone maxillofacial prosthetic materials. Dent Mater 1999;15(06):450-455 